\title{
Use of Cross-Correlation Function to Study Formation Mechanism of Massive Elliptical Galaxies
}

\author{
Tuli De ${ }^{1}$, Tanuka Chattopadhyay ${ }^{2}$ and Asis Kumar Chattopadhyay ${ }^{3,4}$ \\ ${ }^{1}$ Department of Oncology, Novartis Health Care Pvt. Ltd., Hyderabad, India \\ ${ }^{2}$ Department of Applied Mathematics, University of Calcutta, 92 A.P.C Road, Calcutta 700009, India \\ ${ }^{3}$ Department of Statistics, University of Calcutta, 35, B.C.Road, Calcutta 700019, India \\ ${ }^{4}$ Email: akcstat@caluniv.ac.in
}

(Received July 02, 2014; AcCEPTEd October 21, 2014)

\begin{abstract}
Spatial clustering nature of galaxies has been studied previously through auto correlation function. The same type of cross-correlation function has been used in the present work to investigate parametric clustering nature of galaxies with respect to masses and sizes of galaxies. Here, formation and evolution of several components of nearby massive early type galaxies $\left(M_{*} \geq 1.3 \times 10^{11} \mathrm{M}_{\odot}\right)$ have been envisaged through cross-correlations, in the mass-size parametric plane, with high redshift $(0.2 \leq z \leq 7)$ ETGs. It is found that the inner most components of nearby ETGs have significant correlation $(\sim 0.5 \pm(0.02-0.07))$ with ETGs in the highest redshift range $(2 \leq z \leq 7)$ called 'red nuggets' whereas intermediate components are highly correlated $(\sim 0.65 \pm(0.03-0.07))$ with ETGs in the redshift range $0.5 \leq z \leq 0.75$. The outermost part has no correlation in any range, suggesting a scenario through in situ accretion. The above formation scenario is consistent with the previous results obtained for NGC5128 and to some extent for nearby elliptical galaxies after considering a sample of ETGs at high redshift with stellar masses greater than or equal to $10^{8.73} \mathrm{M}_{\odot}$. So the present work indicates a three phase formation instead of two as discussed in previous works.
\end{abstract}

Keywords: clustering - cross-correlation - elliptical galaxies

\section{INTRODUCTION}

In 1934, Hubble observed that the frequency distribution of the count of galaxies over the space is strongly skewed but the distribution of its logarithm is close to symmetric. Bok (1934) and Mowbray (1938) found that variance of the count is considerably larger than expected for a random galaxy distribution. Such studies indicates that locally galaxies are clustered over space. Several attempts have been made to study this clustering nature on the basis of angular positions of the galaxies. Most of them (Chandrasekhar \& Munch 1952; Zwicky 1953; Limber 1953, 1954) have used spatial and angular correlation functions to study this phenomenon. In this area, the contributions of Neyman, Scott, \& Shane (1954) is very significant. This spatial clustering nature motivated us to investigate the clustering nature with respect to the other parameters also by using the same approach.

Classical formation of elliptical galaxies can be divided into five major categories, e.g. (i) the monolithic collapse model (Larson 1975; Carlberg 1984; Arimoto \& Yoshii 1987), (ii) the major merger model (Toomre \& Toomre 1972; Ashman \& Zepf 1992; Zepf et al. 2000; Bernardi et al. 2011;
Prieto et al. 2013), (iii) the multiphase dissipational collapse model (Forbes, Bordie, \& Grillmair 1997), (iv) the dissipationless merger model (Bluck, Conselice, \& Buitrago 2012; Newman et al. 2012), and (v) the accretion and in situ hierarchical merging (Mondal, Chattopadhyay, \& Chattopadhyay 2008). Recent observations in the deep field have explored that high redshift galaxies have the size of the order of 1 kpc (Daddi, Renzini, \& Pirzkal 2005; Trujillo, Feulner, \& Goranova 2006; Damjanov, Abraham, \& Glazebrook 2011) and have higher velocity dispersion (Cappellari, di Serego Alighieri, \& Cimatti 2009; Onodera et al. 2012) than nearby early type galaxies (ETGs) of the same stellar mass. Galaxies at intermediate redshifts (since $z \approx 2.5$ ) have stellar masses and sizes increased by a factor almost 3-4 (van Dokkum, Whitaker, \& Brammer 2010; Papovich, Bassett, \& Lotz 2012; Szomoru, Franx, \& van Dokkum 2012). All these evidences suggest that massive ETGs form in two phases viz. inside-out, i.e. intense dissipational process like accretion (Dekel, Sari, \& Ceverino 2009) or major merger form, an initially compact inner part. After this a second slower phase starts when the outermost part is developed through non-dissipational process, e.g. dry minor merger. The above development arising 
both in the field of observations as well as theory, severely challenges classical models like, monolithic collapse or major merger and favours instead a 'two phase' scenario (Oser et al. 2010; Johansson, Naab, \& Ostriker 2012) of the formation of nearby elliptical galaxies. The task remains is to check whether the compact inner parts of the nearby ETGs have any kind of similarity with the fossil bodies (viz. 'red nuggets') at high redshift.

In a previous work (Huang et al. 2013b), the authors have pursued the above task through matching 'median' values of the two systems. They used this measure with respect to univariate data and the univariate data they considered, are either stellar 'mass' or 'size'. For ETGs in the redshift range, $0.5 \leq z \leq 7$, considered, in the present data set, the stellar mass-size correlation is $r\left(M_{*}, R_{\mathrm{e}}\right)=0.391, p$-value $=0.00$. For nearby ETGs for inner, intermediate and outer components the stellar mass-size correlations with $p$-values are $r\left(M_{*}, R_{\mathrm{e}}\right)=0.720, p$-value $=0.00, r\left(M_{*}, R_{\mathrm{e}}\right)=0.636$, $p$-value $=0.00, r\left(M_{*}, R_{\mathrm{e}}\right)=0.573, p$-value $=0.00$, respectively and all these values are highly significant. Hence, use of univariate median matching is not sufficient in the present context for highly correlated bivariate data. Also, median does not include all objects in a particular data set. For this a more sophisticated technique is in demand for such kind of investigation.

In the present work, we have used the mass-size data of high redshift galaxies and nearby ETGs and used a cross correlation, especially designed to study bivariate data. This is more trustworthy and meaningful in the present situation. In Section 2, we have discussed the data set and in Section 3, we have described the method. The results and interpretations are given under Section 4.

\section{DATA SETS}

We have considered eight data sets. Data sets 1-3 have been taken from Ho et al. (2011). In data sets 1-3 there are 70 nearby ETGs and corresponding to each massive ETG, there are mass-size data for (i) an inner component with effective radii $R_{\mathrm{e}} \leq 1 \mathrm{kpc}$, (ii) an intermediate component with effective radii $R_{\mathrm{e}} \sim 2.5 \mathrm{kpc}$, and (iii) an outer envelope with $R_{\mathrm{e}} \sim 10 \mathrm{kpc}$ (Huang et al. 2013a). Data sets 4-8 consist of mass-size data of ETGs in the high redshift zone (viz. $0.5<z \leq 2.7$ ) and their masses have the lower limit $M_{*} \geq 10^{8.73} \mathrm{M}_{\odot}$. The entire redshift zone has been divided into five redshift bins which are, $0.5<z \leq 0.75,0.75<$ $z \leq 1,1<z \leq 1.4,1.4<z \leq 2.0,2.0<z \leq 2.7$ similar to Huang et al. (2013b) but unlike Huang et al. (2013b) we also included intermediate-mass high-redshift galaxies. Data sets $4-8$ contains 786 high-redshift ETGs from the following works.

392 galaxies $(0.2 \leq z \leq 2.7)$ from Damjanov et al. (2011), $32(1.5<z<3)$ galaxies from GOODS-NICMOS survey (Conselice et al. 2011) for Sérsic (1968) index $n>2,21$ galaxies from CANDELS (Grogin, Kocevski, \& Faber 2011) $(1.5<z<2.5), 107$ from Papovich et al. (2012)(1.5 $\leq z \leq$
Table 1. Multivariate multi sample test for the matching of parent distributions corresponding to data sets $4-8$ (at $0.5 \%$ level of significance).

\begin{tabular}{lcll}
\hline \hline Sample1 & Sample2 & $p$-value & Decision \\
\hline Damjanov & Grogin et al. (2011) & 0.005 & Accepted \\
et al.(2011) & Conselice et al. (2011) & 0.007 & Accepted \\
,, & Nilsson et al. (2013) & 0.0447 & Accepted \\
,, & Mclure et al. (2012) & 0.003 & Accepted \\
,, & Saracco et al. (2011) & 0.096 & Accepted \\
,, & Papovich et al. (2012) & 0.000 & Rejected \\
\hline \hline
\end{tabular}

2.5), 48 from Mclure, Pearce, \& Dunlop (2012) $(1.3<z<$ 1.5), 62 from Saracco, Longhetti, \& Gargiulo (2011) $(1<$ $z<2$ ), 124 galaxies from Nilsson et al. (2013).

Since the data sets are chosen from different sources, they have various selection biases and errors, etc. Hence, to judge their compatibility we have performed a multivariate multi sample matching test (Puri \& Sen 1966; Mckean, Biedermann, \& Büger 1974; Mondal et al. 2008) to see whether they have the same parent distribution or not. From previous works, it is evident that galaxies have undergone cosmological evolution via merger or accretion (Khochfar \& Silk 2006; De Lucia \& Blaziot 2007; Guo \& White 2008; Kormendy et al. 2009; Hopkins, Carton, \& Bundy 2010; Naab 2013) and we have performed the matching test for galaxies within the same redshift zone. The data set taken from Damjanov et al. (2011) contains maximum number of galaxies within the entire redshift zone $(0.2 \leq z \leq 2.7)$ used in the present analysis. For this we have compared it with the other sets. The results are given in Table 1. It is clear from Table 1 that all the tests are accepted except one (Papovich et al. 2012) where the matching redshift zone is very narrow. Since almost in $99 \%$ cases, the test is accepted we assume that the data set consisting of samples from different sources is more or less homogeneous with respect to mass-size plane.

For testing completeness of the combined data sets $4-8$, we have plotted the data points in the size-mass plane (viz. Figure 1). Then we have performed $V / V_{\max }$ test. This test was first used by Schmidt (1965) to study the space distribution of a complete sample of radio quasars from $3 \mathrm{eR}$ catalogue. According to the test, let $F_{m}$ be the limiting flux of a survey. We define two columns $V(r)=\frac{4 \pi r^{3}}{3}$ and $V_{\max }=\frac{4 \pi r_{m}^{3}}{3}$, where $r$ is the radial distance to a quasar and $r_{m}$ is the limiting distance at which flux of a quasar with luminosity $L$ reduces to $r_{m}$. For $r>r_{m}$ the quasars do not belong to the sample under consideration. If the quasars are expected to be uniformly distributed then $V / V_{\max }$ are uniformly distributed over $[0,1]$. Then $\left\langle V / V_{\max }\right\rangle=0.5$. According to the above theory, we have computed $\left\langle\log R_{e} / \log R_{e, \max }\right\rangle$ for the combined data set and it is $\sim 0.3$, i.e. the combined data set of high redshift galaxies is complete up to an accuracy of almost $70 \%$. For comparison we have also plotted the combined data sets 1-3, in the size-mass plane (viz. Figure 2). 


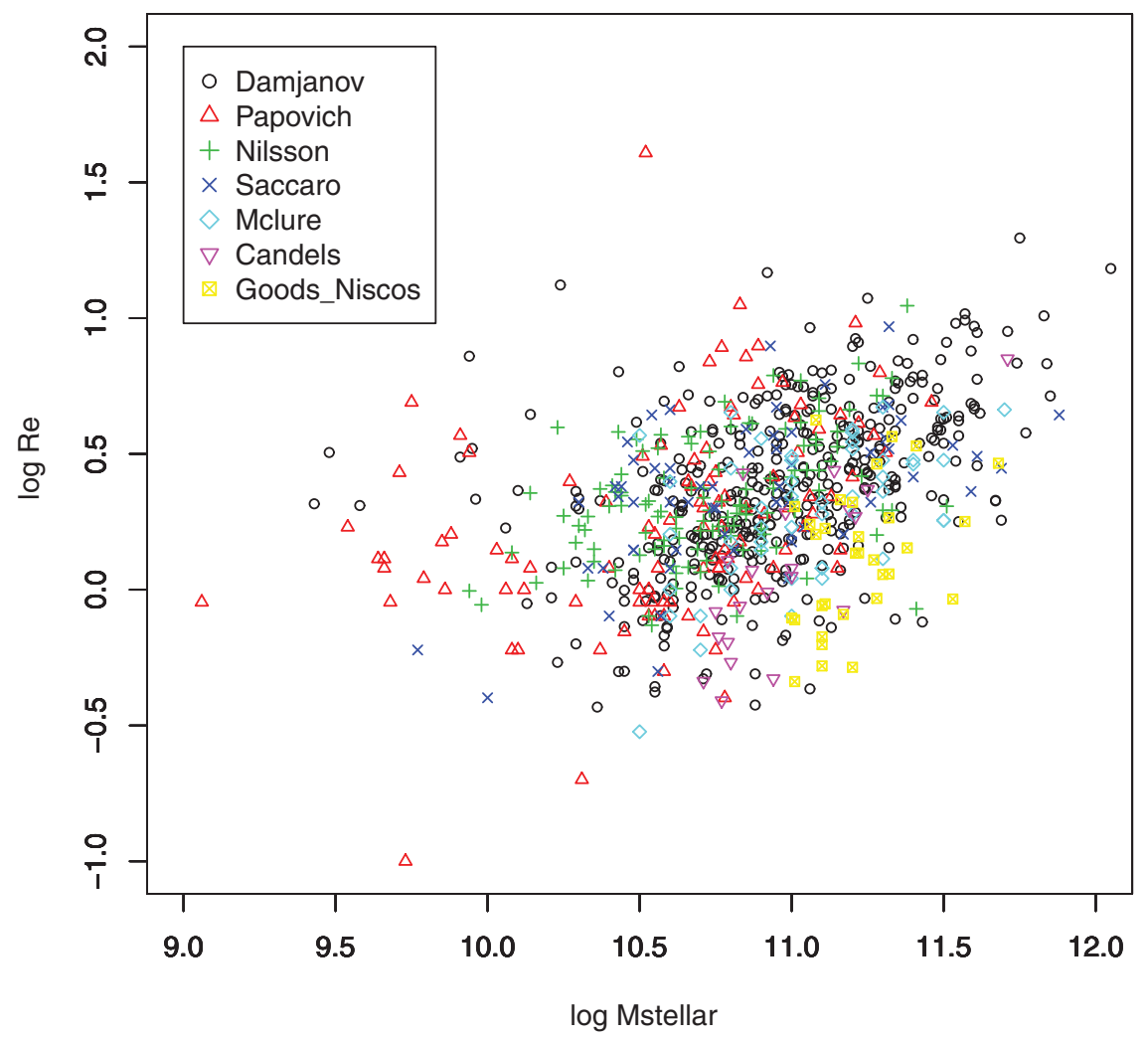

Figure 1. $\log R_{e}$ versus $\log M$ plot of the data points for data sets 4-8.

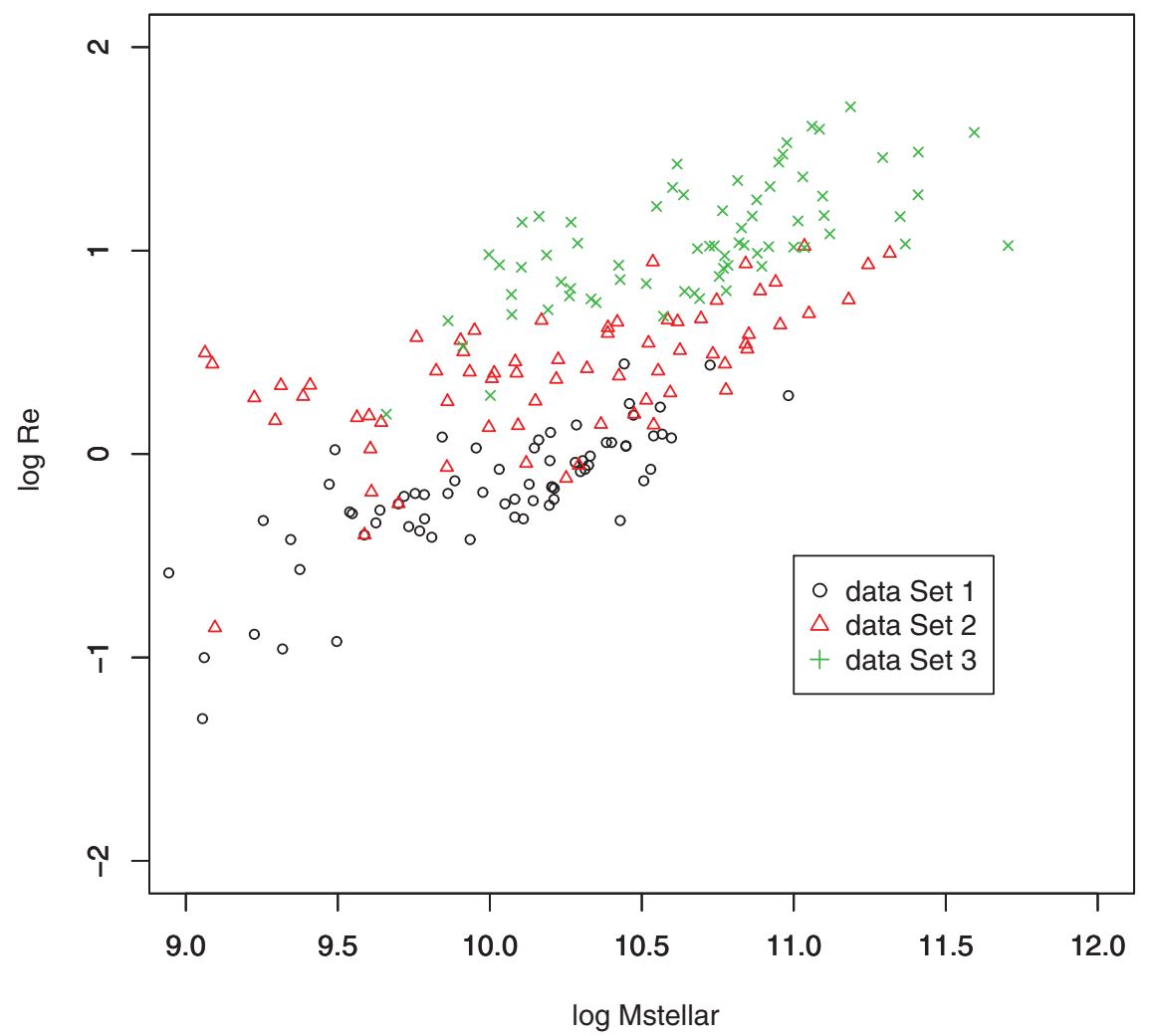

Figure 2. $\log R_{e}$ versus $\log M$ plot of the data points for data sets 1-3. 


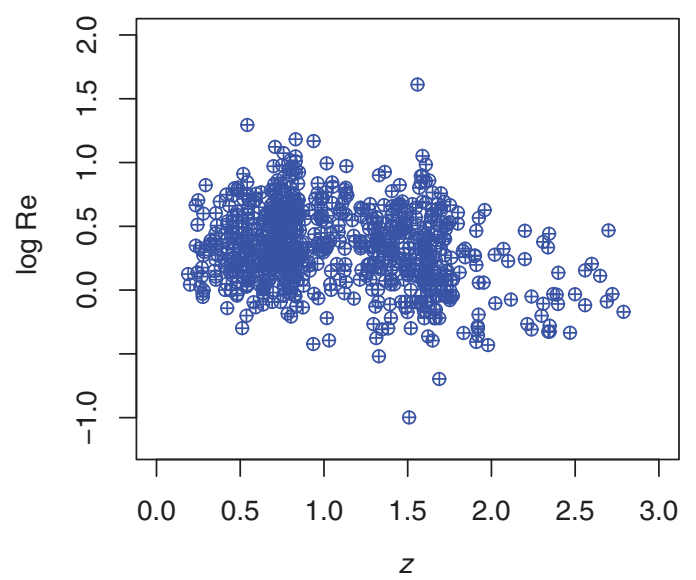

Figure 3. Logarithm of the effective radius versus redshift plot for the entire sample of ETGs in $0.2 \leq z \leq 2.7$.

It is to be noted that in Ho et al. (2011) paper, the magnitude values of the three components corresponding to each ETG are given from which, luminosities are computed. Then these luminosities are multiplied by $(M / L)$ ratios for obtaining stellar masses. The $(M / L)$ ratios are computed following Bell $\&$ de Zong (2001). We have not been able to retrieve data for some high redshift galaxies and instead included some new data from other recent references so that sample size of high redshift galaxies are somewhat reduced in our case, but the overall distribution of these galaxies are similar in the size-redshift plane with those considered by Huang et al. (2013b) (viz. Figure 1 of Huang et al. (2013) and Figure 3 in the present work) except the region $1 \leq z \leq 2$ which is more populated than Huang et al. (2013b) sample as we have included new galaxies in data sets $4-8$.

\section{METHOD}

The theory of the special distribution of galaxies has been discussed by several authors like Peebles (1980), Blake et al. (2006), Martinez \& Saar (2002), etc. During 1950s, the most extensive statistical study was performed by Neyman and Scott. Their work was based on the large amount of data obtained from the LICK survey. The main empirical statistics they used were the angular auto correlation function of the galaxy counts (Neyman, Scott, \& Shane 1956) and Zwicky's index of clumpiness (Neyman, Scott, \& Shane 1954).

Neyman \& Scott (1952) introduced this theory on the basis of four assumptions viz. (i) galaxies occur only in clusters, (ii) the number of galaxies varies from cluster to cluster subject to a probabilistic law, (iii) the distribution of galaxies within a cluster is also subject to a probabilistic law, and (iv) the distribution of cluster centres in space is subject to a probabilistic law described as quasi-uniform. As the observed distribution of number of galaxies does not follow Poisson law, it is suspected that not only the apparent but also the actual spatial distribution of galaxies is clustered.
In the present work, attempts have been made to establish the same postulates with respect to mass-size distribution of galaxies. Here the hypothesis is 'there is clustering nature also in the galaxy distribution with respect to the parameters mass and size of the galaxies'. This particular hypothesis also has been studied by several authors. But we have followed the same approach as that used to establish spatial clustering as discussed above.

In cosmology the cross-correlation function $\xi(r)$ of a homogeneous point process is defined by

$$
\mathrm{d} P_{12}=\pi^{2}[1+\xi(r)] \mathrm{d} V_{1} \mathrm{~d} V_{2},
$$

where $r$ is the separation vector between the points $x_{1}$ and $x_{2}$ and $\pi$ is mean number density. Considering two infinitesimally small spheres centred in $x_{1}$ and $x_{2}$ with volumes $\mathrm{d} V_{1}$ and $\mathrm{d} V_{2}$, the joint probability that in each of the spheres lies a point of the point process is as follows:

$$
\mathrm{d} P_{12}=\lambda_{2}\left(x_{1}, x_{2}\right) \mathrm{d} V_{1} \mathrm{~d} V_{2} \text {. }
$$

In (2), $\lambda_{2}\left(x_{1}, x_{2}\right)$ is defined as the second order intensity function of a point process.

If the point field is homogeneous, the second-order intensity function $\lambda_{2}\left(x_{1}, x_{2}\right)$ depends only on the distance $r=\left|x_{1}-x_{2}\right|$ and direction of the line passing through $x_{1}$ and $x_{2}$. If, in addition, the process is isotropic,the direction is not relevant and the function only depends on $r$ and may be denoted by $\lambda_{2}(r)$. Then

$$
\xi(r)=\frac{\lambda_{2}(r)}{\pi^{2}}-1 .
$$

Different authors proposed several estimators of $\xi(r)$. Natural estimators have been proposed by Peebles \& Hauser (1974). The cross-correlation function $\xi(r)$ can be estimated from the galaxy distribution by constructing pair counts from the data sets. A pair count between two galaxy populations 1 and 2, $D_{1} D_{2}(r)$, is a frequency corresponding to separation $r$ to $r+\delta r$ for a bin of width $\delta r$ in the histogram of the distribution $r, D_{i} R_{j}$ and $R_{i} R_{j}$ denote the same pair counts corresponding to one galaxy sample and two simulated samples respectively, $i, j=1,2$. Two natural estimators are given by

$$
\begin{aligned}
& \hat{\xi_{1}}=\frac{D_{1} D_{2}(r)}{D_{2} R_{1}(r)}-1, \\
& \hat{\xi_{2}}=\frac{D_{1} D_{2}(r)}{D_{1} R_{2}(r)}-1 .
\end{aligned}
$$

Another two improved estimators are Blake et al. (2006)

$$
\hat{\xi_{3}}=\frac{D_{1} D_{2}(r) R_{1} R_{2}(r)}{D_{1} R_{2}(r) D_{2} R_{1}(r)}-1,
$$

and

$$
\hat{\xi_{4}}=\frac{D_{1} D_{2}(r)-D_{1} R_{2}(r)-D_{2} R_{1}(r)+R_{1} R_{2}(r)}{R_{1} R_{2}(r)} .
$$

The first two estimates are potentially biased. As in the present situation, we are considering mass-size parametric space, we have taken $r$ as the Euclidean distance between two (mass-size) points of two galaxies, either original or 
simulated. In order to generate simulated samples of mass and size, we have used uniform distribution of mass and size with ranges selected from original samples. Here, $r$ is normalised by dividing the original separation by the maximum separation.

\subsection{Simulation and computation}

In order to determine the cross-correlation function by incorporating the effects of the statistical fluctuations, we have generated random unclustered realisations (denoted by $R_{i}$ ) of the mass-size distribution in the same range as that of the corresponding observed samples (denoted by $D_{i}$ ).

The simulation and computation steps are as follows:

(i) Select one pair of observed bivariate (mass-size) samples. These are denoted by $D_{1}$ and $D_{2}$.

(ii) Corresponding to $D_{1}$, determine the minimum and maximum values of masses and sizes, respectively. Let the values be $\left(m_{\min }, m_{\max }\right)$ and $\left(s_{\min }, s_{\max }\right)$, respectively.

(iii) Assuming uniform distribution of masses over the range $m_{\min }<m<m_{\max }$, generate one mass at random. Let it be $m_{1}$. Similarly assuming uniform distribution over the range $s_{\min }<s<s_{\max }$, generate one size at random. Let it be $s_{1}$. Then $\left(m_{1}, s_{1}\right)$ will give the first paired observation for the simulated sample $R_{1}$.

(iv) Repeat step (iii), a large number of times in order to generate a large number of paired values for the simulated sample $R_{1}$.

(v) Repeat the steps (ii)-(iv) for $D_{2}$ in order to generate $R_{2}$.

(vi) After computing the Euclidean distances between pairs $\left(D_{i} R_{j}\right), i, j=1,2$ and construction of histograms, compute the cross-correlation function by using formulae (6) and (7) in order to find estimator 1 and estimator 2.

(vii) Repeat steps (i)-(iv) by considering different pairs of (mass-size) samples.

(viii) To compute the standard errors of estimators use boot strap method.

\section{RESULTS AND DISCUSSION}

We have computed the cross-correlation functions of each of data sets $1-3$ with data sets $4-8$, i.e. we have tried to find any kind of correlation between three components of nearby ETGs with high redshift ETGs in five redshift bins as mentioned above. We have found significant correlation between data sets 1 and 8 and between data sets 2 and 4 . This is clear from Figures 4 and 5, respectively where the correlations are as high as $0.5 \pm(0.02-0.07)$ and $0.65 \pm(0.03-0.07)$ for both the estimates at minimum separation (viz.r $\sim 0.1$ ). These show that the innermost components of nearby elliptical galaxies are well in accordance with highest redshift mas-

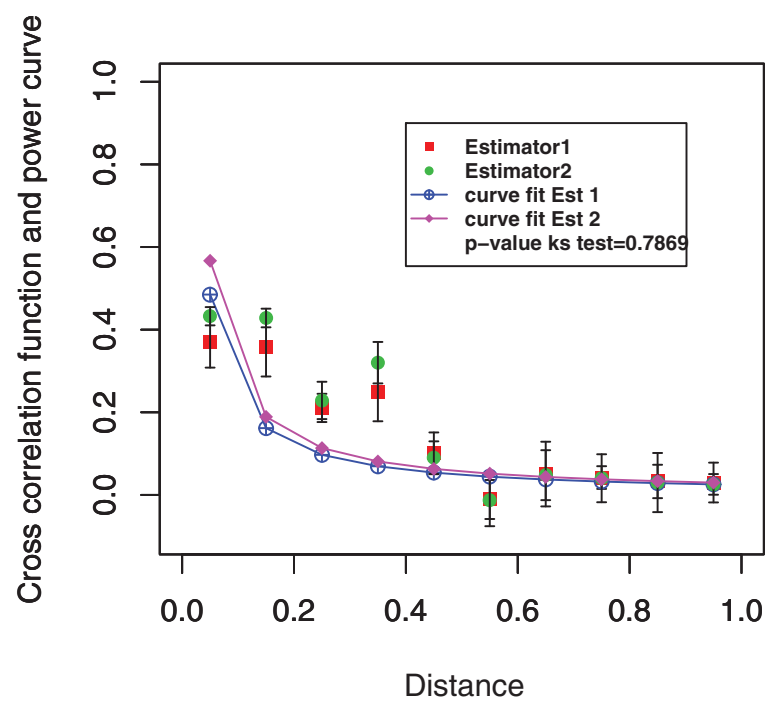

Figure 4. Cross-correlation function $\xi(r)$ versus normalised distance bin $r$ between data sets 1 and 8 . The solid lines are power laws for both the estimators as $\xi(r) \propto \frac{1}{r}$.

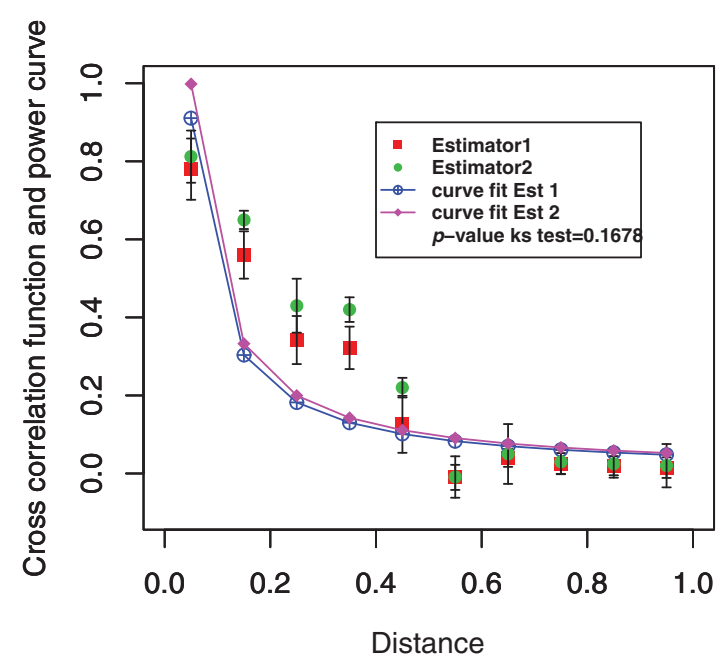

Figure 5. Cross-correlation function $\xi(r)$ versus normalised distance bin $r$ between data sets 2 and 4 . The solid lines are power law fits for both the estimators as $\xi(r) \propto \frac{1}{r}$.

sive ETGs (viz. mass $\sim 10^{11.14} \mathrm{M}_{\odot}$ and $R_{\mathrm{e}} \sim 0.92 \mathrm{kpc}$ ), known as 'red nuggets' but the intermediate components are highly correlated with galaxies in the redshift bin $0.5 \leq z \leq 0.75$ having median mass and size, $10^{10.87} \mathrm{M}_{\odot}$ and $2.34 \mathrm{kpc}$, respectively. If we merge data sets 1 and 2 and compare with high $z$ galaxies in five redshift bins, the cross-correlation functions are all close to zero at all separations unlike Huang et al. (2013b).

The above result is somewhat consistent with the work of Huang et al. (2013b) in a sense that the inner and intermediate parts are the fossil evidences of high redshift galaxies but unlike Huang et al. (2013b), components 1 and 2 together show no correlation with all high redshift ETGs together and 


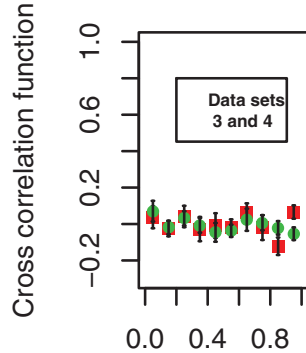

Distance

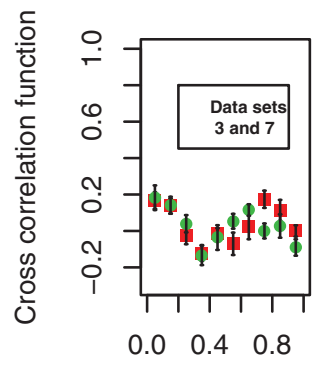

Distance

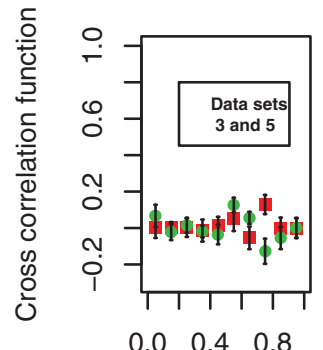

Distance

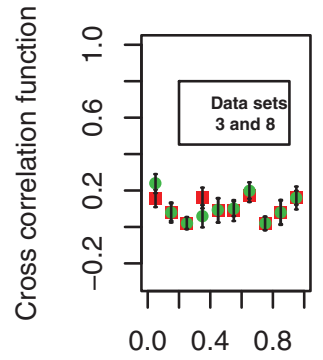

Distance

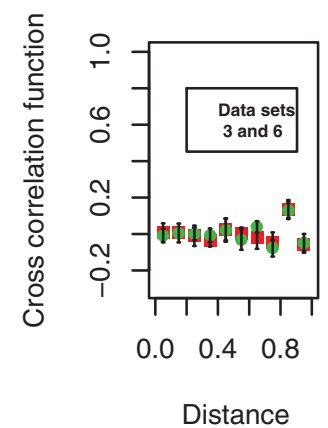

Figure 6. Cross-correlation function $\xi(r)$ versus normalised distance bin $r$ between data set 3 and all redshift bins.

they are highly correlated with high redshift ETGs in two redshift bins. This indicates clearly two different epochs of structure formation as shown by their $z$ values.

After finding the cross-correlation functions between data sets 1 and 8 , we have fitted a power law assuming the relation

$$
\xi(r) \propto \frac{1}{r},
$$

i.e.

$$
\xi(r)=A r^{-1},
$$

where for estimator $1, A=0.02672$ and for estimator 2 , $A=0.031395$.

We have also performed Kolmogorov-Smirnov test (Kolmogorov 1933) for justifying the goodness of fit of the power law. Here fitting of power law helps us to reject a model with no correlation and the KS test indicates the significance level ( $p$-value) which is quite high in the present situation. Here, we have assumed that the cross correlations and the fitted values are samples coming from the distribution function of a Pareto distribution. The $p$-values for this test for estimator 1 is 0.4175 and for estimator 2 is 0.7869 , signifying that the tests are accepted and the fitted power law gives well justification for the cross-correlation and distance relationship. We have fitted similar power laws for cross-correlation function and distance for data sets 2 and 4 . Here, the proportionality constants are 0.0247 for estimator 1 and 0.0369 for estimator 2. The Kolmogorov-Smirnov tests give $p$-values $=0.7869$ for both the estimators, signifying that in this case also the relationship is well justified.
On the other hand, cross-correlation function, computed for data set 3 with galaxies in the above five bins are all insignificant which is clear from Figure 6.

It is well known that major and minor mergers take important role in the formation and evolution of massive elliptical galaxies (Khochfar \& Silk 2006; De Lucia \& Blaizot 2007; Guo \& White 2008; Kormendy et al. 2009; Hopkins et al. 2010; Naab 2013). Generally ellipticals having maximum masses are speculated to be formed at $z \sim 6$ or higher and the environment for their formation is a dissipative one. Subsequently, they become massive $\left(\sim 10^{11} \mathrm{M}_{\odot}\right)$ and compact in a very short interval of time at $z \sim 2$ (Dekel et al. 2009; Oser et al. 2010; Feldmann, Carollo, \& Mayer 2011; Oser et al. 2012). But at the same time a significant fraction remains less active at $z \sim 2$. They are 4-5 times more compact and less massive by a factor of 2 corresponding to their low-redshift descendants (Buitrago et al. 2008; van Dokkum et al. 2008; VanderWel et al. 2008; Cimatti, Cassata, \& Pozzetti 2008; Bezanson et al. 2009; van Dokkum et al. 2010; Whitaker et al. 2012).

For the massive ellipticals in the present sample, the innermost cores (data set 1) are well in accordance with highest redshift $(2.0<z \leq 2.7)$ galaxies and their core masses (viz. median value $\sim 10^{10.203} \mathrm{M}_{\odot}$ and $10^{10.6839} \mathrm{M}_{\odot}$, respectively). Hence, it is reasonable to separate that these high redshift population forms the cores of at least some, if not all, present day massive ellipticals. Thus formation of massive ellipticals only by monolithic collapse model is challenged because they will be too small and too red (van Dokkum et al. 2008; FerréMateu, Vazdekis, \& Trujillo 2012), the subsequent evolution 
forming the intermediate (data set 2) and outer part (data set 3 ) might be as follows. On the aspect of major or minor major, following Naab, Johansson, \& Ostriker (2009) it is seen that if $M_{i}$ and $r_{i}$ be the mass and radius of a compact initial stellar system with a total energy $E_{i}$ and mean square speed $\left\langle v_{i}^{2}\right\rangle$ and $M_{a}, r_{a}, E_{a}$ and $\left\langle v_{a}^{2}\right\rangle$ be the corresponding values after merger with other systems then,

$$
\begin{aligned}
\frac{\left\langle v_{\mathrm{f}}^{2}\right\rangle}{\left\langle v_{i}^{2}\right\rangle} & =\frac{(1+\eta \epsilon)}{1+\eta}, \\
\frac{r_{g, \mathrm{f}}}{r_{g, i}} & =\frac{(1+\eta)^{2}}{(1+\eta \epsilon)}, \\
\frac{\rho_{\mathrm{f}}}{\rho_{i}} & =\frac{(1+\eta \epsilon)^{3}}{(1+\eta)^{5}},
\end{aligned}
$$

where the quantities with suffix ' $\mathrm{f}$ ' are the final values, $\eta=\frac{M_{a}}{M_{i}}, \epsilon=\left\langle v_{a}^{2}\right\rangle /\left\langle v_{i}^{2}\right\rangle, \rho$ is the density. Then for $\eta=1$ (major merger), the mean square speed remains same, the size increases by a factor of 2 and densities drop by a factor of 4. Now, in the present situation, the intermediate part (data set 2) has radii (median value $\left\langle R_{\mathrm{e}}\right\rangle_{2} \sim 2.560 \mathrm{kpc}$ ) which is almost 3 times larger than the radii of inner part $\left(\left\langle R_{\mathrm{e}}\right\rangle \sim\right.$ $0.6850 \mathrm{kpc})$.

Also in a previous work (Chattopadhyay et al. 2009; Chattopadhyay, Mondal, \& Chattopadhyay 2013) on the brightest elliptical galaxy NGC 5128, we have found three groups of globular clusters. One is originated in original cluster formation event that coincided with the formation of elliptical galaxy and the other two, one from accreted spiral galaxy and other from tidally stripped dwarf galaxies. Hence we may conclude from the above discussion that the intermediate parts of massive elliptical is formed via major merger with the high redshift galaxies in $0.5 \leq z<0.75$, whose median mass and size are respectively $10^{10.87} \mathrm{M} \odot \& 2.34 \mathrm{kpc}$, respectively.

In the limit when $\left\langle v_{a}^{2}\right\rangle \ll\left\langle v_{i}^{2}\right\rangle$ or $\epsilon \ll 1$, the size increases by a factor of 4 (minor merger). In the present case, the outermost parts of massive ellipticals have sizes much larger $\left(\left\langle R_{\mathrm{e}}\right\rangle_{3} \sim 10.54 \mathrm{kpc}\right)$, so $\left.\left\langle R_{\mathrm{e}}\right\rangle_{3}-\left\langle R_{\mathrm{e}}\right\rangle_{1} \sim 10 \mathrm{kpc}\right)$ than innermost part. Also, median mass of this part is of the order of $10^{10.6839} \mathrm{M}_{\odot}$ which is comparable to the combined masses of few dwarf galaxies. So, it might be suspected that the outermost part is primarily composed of stellar components of tidally accreted satellite dwarf galaxies. This is also consistent with our previous works (Chattopadhyay et al. 2009, 2013) in case of NGC 5128. Since, data set 3 has no correlations with any subset of high redshift galaxies, we cannot specifically confirm their formation epoch but we can atmost say that their formation process is different from the innermost and intermediate part.

Finally, we can conclude that formation of nearby massive ellipticals have three parts, inner, intermediate, and outermost, whose formation mechanisms are different. The innermost parts are descendants of high ETGs called 'red nuggets'. The innermost parts are formed by major mergers with tidally stripped satellite dwarf galaxies (Mondal et al. 2008; Chattopadhyay et al. 2009, 2013; Mihos et al. 2013). Since, the densities and velocity dispersion values and abundances are not available with the present data sets, so more specific conclusions can be drawn if these data are available for massive ellipticals and satellite dwarfs. But at this moment we can say, that since two different formation scenarios are very unlikely for the same galaxy at a particular epoch, so the above study is indicative of a 'third phase' of formation of the outermost parts of massive nearby ellipticals rather than a 'two phase one' as indicated by previous authors.

\section{ACKNOWLEDGEMENTS}

The authors are grateful to the referee for suggestions in improving the quality of the work.

\section{References}

Arimoto, N., \& Yoshii, Y. 1987, A\&A, 173, 23

Ashman, K. M., \& Zepf, S. E. 1992, ApJ, 384, 50

Bell, E. F., \& de Zong, R. S. 2001, ApJ, 550, 212

Bernardi, M., Roche, N., Shankar, F., \& Sheth, R. K. 2011, MNRAS, 412, L6

Bezanson, R., et al. 2009, ApJ, 697(2), 1290

Blake, C., Pope, A., Scott, D., \& Mobasher, B. 2006, MNRAS, 368, 732

Bluck, A. F. L., Conselice, C. J., \& Buitrago, F. 2012, ApJ, 747, 34

Bok, B. J. 1934, Bull. Harv. Obser., 895, 1

Buitrago, F., Trujillo, I., Conselice, C.J., et al. 2008, ApJ, 687, L61

Cappellari, M., di Serego Alighieri, S., \& Cimatti, A. 2009, ApJ, 704, L34

Carlberg, R. G. 1984, ApJ, 286, 403

Chandrasekhar, S., \& Munch, G. 1952, ApJ, 115, 103

Chattopadhyay, A. K., Mondal, S., \& Chattopadhyay, T. 2013, CSDA, 57, 17

Chattopadhyay, A. K., et al. 2009, ApJ, 705, 1533

Cimatti, A., Cassata, P., \& Pozzetti, L. 2008, A\&A, 482(1), 21

Conselice, C. J., et al. 2011, MNRAS, 413, 80

Daddi, E., Renzini, A., \& Pirzkal, N. 2005, ApJ, 626, 680

Damjanov, I., Abraham, R. G., \& Glazebrook, K. 2011, ApJ, 739, L44

Dekel, A., Sari, R., \& Ceverino, D. 2009, ApJ, 703, 785

De Lucia, G., \& Blaizot, J. 2007, MNRAS, 375(1), 2

Feldmann, R., Carollo, C. M., \& Mayer, L. 2011, ApJ, 736(2), 11

Ferré-Mateu, A., Vazdekis, A., \& Trujillo, I. 2012, MNRAS, 423(1), 632

Forbes, D. A., Bordie, J. P., \& Grillmair, C. J. 1997, ApJ, 113, 1652

Grogin, N. A., Kocevski, D. D., \& Faber, S. M. 2011, ApJS, 197, 35

Guo, Q., \& White, S. D. M. 2008, MNRAS, 384(1), 2

Ho, L. C., et al. 2011, ApJ, 197, 21

Hopkins, P. F., Carton, D., \& Bundy, K. 2010, ApJ, 724, 915

Huang, S., Ho, L. C., \& Peng, C. Y. 2013a, ApJ, 766

Huang, S., Ho, L. C., \& Peng, C. Y. 2013b, ApJ, 768, L28

Johansson, P. H., Naab, T., \& Ostriker, J. P. 2012, ApJ, 754, 115

Khochfar, S., \& Silk, J. 2006, ApJ, 648(1), L21

Kolmogorov, A. 1933, Ist. Ital. Attuari, 4, 83

Kormendy, J., et al. 2009, ApJS, 182(1), 216 
Larson, R. B. 1975, MNRAS, 173, 671

Limber, D. N. 1953, ApJ, 117, 134

Limber, D. N. 1954, ApJ, 119, 655

Martinez, V. J., \& Saar, E. 2002, Statistics of the Galaxy Distribution (Boca Raton, Fl: Chapman \& Hall/CRC Press)

McKean, D. C., Biedermann, S., \& Büger, H. 1974, AcSpA, 30(3), 845

McLure, R. J., Pearce, H. J., \& Dunlop, J. S. 2012, MNRAS, submitted (arXiv: 1205.4058)

Mihos, J. C., et al. 2013, ApJ, 762(2), 82.

Mondal, S., Chattopadhyay, T., \& Chattopadhyay, A. K. 2008, ApJ, 683,172

Mowbray, A. G. 1938, PASP, 50, 275

Naab, T. 2013, Modelling the formation of today's massive ellipticals, in IAU Symposium no. 295, ed. D. P. Pasquali, \& A. Q. Ferreras

Naab, T., Johansson, P. H., \& Ostriker, J. P. 2009, ApJ, 699(2), L178

Newman, A. B., et al. 2012, ApJ, 746, 162

Neyman, J., \& Scott, E. L. 1952, ApJ, 116, 144

Neyman, J., Scott, E. L., \& Shane, C. D. 1954, ApJS, 1, 269

Neyman, J., Scott, E. L., \& Shane, C. D. 1956, in Proceedings of the 3rd Berkeley Symposium on Mathematical Statistics and Probability, Vol. 252, 75

Nilsson, M. K. M., et al. 2013, LITHOS, 174, 255

Onodera, M., Renzini, A., \& Carollo, M. 2012 ApJ, 755, 26

Oser, L., et al. 2010, ApJ, 725, 2312
Oser, L., et al. 2012, ApJ, 744(1), 63

Papovich, C., Bassett, R., \& Lotz, J. M. 2012, ApJ, 750, 93

Peebles, P. J. E. 1980, The Large Scale Structure of the Universe (Princeton: Princeton University Press)

Peebles, P. J. E., \& Hauser, M. G. 1974, ApJS, 28, 19.

Prieto, M., et al. 2013, MNRAS, 428, 999.

Puri, M. L., \& Sen, P. K. 1966, Sankhya A, 28(4), 353.

Saracco, P., Longhetti, M., \& Gargiulo, A. 2011, MNRAS, 412, 2707.

Schmidt, M. 1965, in Star and Stellar Systems, Galactic Structures, ed. A. Blaauw, \& M. Schmidt (Vol. 5; Chicago: University of Chicago Press), 513

Sérsic, J. L. 1968, Atlas de Galaxies Australes (Cordoba: Observatoria Astronomico)

Szomoru, D., Franx, M., \& van Dokkum, P. G. 2012, ApJ, 749, 121

Toomre, A., \& Toomre, J. 1972, ApJ, 178, 623

Trujillo, I., Feulner, G., \& Goranova, Y. 2006, MNRAS, 373, L36

van der Wel, A., Rix, H. -W., Wuyts, S., et al. 2011, ApJ, 730, 38

van Dokkum, P. G. 2008, ApJ, 674(1), 29

van Dokkum, P. G., Franx, M., Kriek, M. et al. 2008, ApJL, 677, L5

van Dokkum, P. G., Whitaker, K. E., \& Brammer, G. 2010, ApJ, 709, 1018

Whitaker, K. E., et al. 2012, ApJ, 754(2), L29

Zepf, S. E., et al. 2000, ApJ, 120, 2928

Zwicky, F. 1953, AcHPh, 26, 241 